\title{
Mitteilungen der Deutschen Migräne- und Kopfischmerzgesellschaft e. V.
}

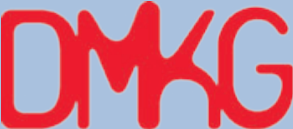

Deutsche Migräne- und Kopfschmerzgesellschaft

\section{Preisverleihung}

Alle 2 Jahre wird von der Deutschen Migräne- und Kopfschmerzgesellschaft (DMKG e. V.) der Wolffram-Preis für Kopfschmerzforschung verliehen. Die 3 Preisträger konnten ihre Arbeiten in internationalen Fachzeitschriften veröffentlichen und lieferten so interessante Beiträge auf dem Gebiet der Kopfschmerzforschung.

Der erste Preis mit 5000 Euro ging an Katharina Kamm aus München. Sie ist Assistenzärztin der Neurologischen Klinik und Poliklinik des Klinikums der Universität München in Großhadern. Sie konnte zeigen, dass sowohl Migräne- als auch Clusterkopfschmerzpatienten bereits außerhalb von Kopfschmerzattacken gegenüber gesunden Kontrollen erhöhte Spiegel des Schmerzbotenstoffes Calcitonin Gene Related Peptide (CGRP) in der Tränenflüssigkeit aufweisen. Die Einnahme einer Akutmedikation führt zur Normalisierung dieser Werte in der Tränenflüssigkeit. Dies zeigt, dass die Bestimmung von CGRP in der Tränenflüssigkeit eine einfache und verlässliche Methode ist, um das Neuropeptid bei Kopfschmerzpatienten nachzuweisen. Bislang konnte CGRP nur in Venenblut gemessen werden. „Diese Arbeit wurde als preiswürdig erachtet, da sie im Alltag eine Bestimmung von CGRP aus der Tränenflüssigkeit erlaubt, die leicht durchführbar, schnell zu erlernen ist und gut toleriert wird“, so PD Dr. Tim Jürgens, Präsident der DMKG e. V.

Den zweiten Preis mit 2500 Euro erhielt Dr. phil. Timo Klan, Psychotherapeut am Psychologischen Institut der Johannes Gutenberg-Universität Mainz. Er stellte in seiner Arbeit ein Testsystem zur psychometrischen Beurteilung von Menschen mit Clusterkopfschmerz vor. Durch die als Selbstbeurteilungsfragebogen konzipierten „Clusterkopfschmerz-Skalen “ ist eine umfassende und für den Clusterkopfschmerz spezifi- sche Einschätzung relevanter psychosozialer Faktoren wie Beeinträchtigung und Krankheitsbewältigung möglich, die über die Messung der Kopfschmerzaktivität hinausgeht. „Damit können therapeutische Interventionen im klinischen Alltag, aber auch in wissenschaftlichen Studien noch besser beurteilt werden, sodass Behandlungsmaßnahmen besser geplant werden können“, so Dr. Thomas Dresler, Psychologe am Universitätsklinikum Tübingen und Leiter der Begutachtungskommission der DMKG.

Der dritte Preis mit 1000 Euro wurde an Hanna Sophie Lapp verliehen, Assistenzärztin an der Klinik und Poliklinik für Neurologie des Universitätsklinikums Dresden. Sie untersucht in ihrer Arbeit erstmals eine spezielle Form der Berührungswahrnehmung mittels sogenannter C-taktiler berührungsempfindlicher Nervenfasern bei Patienten mit Migräne. Lapp konnte zeigen, dass bei Migränepatienten eine veränderte Gewöhnung (Habituation) für C-taktile Reize vorliegt. Als ursächlich werden eine zentrale und periphere Überempfindlichkeit (Sensitisierung) gegenüber diesen mechanisch taktilen Reizen diskutiert. Diese klinische Studie unterstützt die These der veränderten Reizwahrnehmung von Patienten mit Migräne und erweitert diese Theorie um die C-taktile Komponente. „Dies legt nahe, dass Patienten mit Migräne aufgrund ihrer Erkrankung ein verändertes Berührungs- und Kommunikationserleben haben und bisher unbekannte und noch zu untersuchende soziokulturelle Folgen davontragen könnten“, so Jürgens.

\section{WWW.DMKG.DE}

\section{www.attacke-kopfschmerzen.de}

Die Deutsche Migräne- und Kopfschmerzgesellschaft e. V. (DMKG) ist seit 1979 die interdisziplinäre wissenschaftliche Fachgesellschaft für Kopf- und Gesichtsschmerzen, in der Ärzte, Psychologen, Physiotherapeuten, Pharmakologen und Apotheker organisiert sind. Der unabhängige und gemeinnützige Verein setzt sich für die Verbesserung der Therapie der vielen Millionen Patienten in Deutschland mit akuten und chronischen Kopfschmerzen ein. Die Fachgesellschaft fördert die Forschung und organisiert Fortbildungen für medizinische Fachberufe sowie einmal jährlich den Deutschen Schmerzkongress gemeinsam mit der Deutschen Schmerzgesellschaft. Die DMKG ist Mitglied in der Deutschen Gesellschaft für Neurologie (DGN) und in der Weltkopfschmerzgesellschaft (International Headache Society).

\section{IMPRESSUM}

\section{Redaktionelle Betreuung}

Prof. Dr. med. Dr. phil. Stefan Evers Neurologische Klinik

Krankenhaus Lindenbrunn

Am Lindenbrunn 1, 31863 Coppenbrügge

everss@uni-muenster.de

\section{Kontaktadresse der DMKG}

Generalsekretär und Pressesprecher Priv.-Doz. Dr. Charly Gaul Migräne und Kopfschmerzklinik Königstein, Ölmühlweg 31

61462 Königstein am Taunus info@dmkg.de,www.dmkg.de

Die Verantwortung für namentlich gekennzeichnete Artikel liegen bei den jeweiligen Autoren. 